\title{
Waterproof Sensor System for Simultaneous Pressure and Hot-Film Flow Measurements
}

\author{
Martin Schwerter ${ }^{1}$, Monika-Leester-Schädel, Andreas Dietzel \\ Institute of Microtechnology TU Braunschweig, Alte Salzdahlumer Str. 203, 38124 Braunschweig, \\ Germany
}

\begin{abstract}
For simultaneous measurement of pressure and near surface flow conditions allowing indirect determination of wall shear stress in experimental water tunnel environment an integrated hybrid sensor system has been developed. In contrast to known approaches, which are limited to the use in gas atmosphere due to protruding electrical and fragile parts, our sensor system is waterproof shielded and embedded in epoxy resin. Furthermore an amplification circuit for the pressure signal based on a programmable gain amplifier is integrated in direct vicinity to the pressure sensor in order to minimize noise by electromagnetic disturbances. Also sensor systems with on-board digitalization of the pressure signal for direct digital read-out were realized. We present all aspects of system assembly and embedding to one waterproof module. Furthermore, read-out strategies as well as sensor test results in air and water are shown and watertightness is confirmed.
\end{abstract}

Keywords: pressure sensor; hot-film sensor; embedded sensor system; waterproof; integrated electronics; FPGA signal processing

\section{INTRODUCTION}

The importance of air traffic increases in a more and more globalized word. The International Air Transport Association (IATA) predicts an average annual growth for the number of passengers of $3.8 \%$ up to 2034 [1]. In order to reduce the impact of the increasing flight traffic on residents in airport-near urban areas different technologies for noise and pollution reduction are developed [2]. Among these an active high-lift system with a highly curved Coanda flap including feedback from flow and pressure sensing is investigated, which shall help reducing airport approach speeds and noise, thereby improving aircraft compatibility to smaller airports with shorter take-off and landing fields [3]. The active high-lift system is investigated within water tunnel experiments in which information about flow condition shall be obtained by pressure and hot-film sensors and serves as input for the closed loop control. By using water instead of air (classic wind tunnel) both the control frequency and the free-stream velocity are reduced by a factor of around 10 . But sealing and electrical isolation of flush-mounted sensors are challenging. Further the airfoil model is scaled down to a chord length of $300 \mathrm{~mm}$ and is made of stainless steel in order to withstand the stress in the water tunnel [4]. These geometric restrictions only allow a shallow notch with a cross section of $10 \mathrm{~mm} \cdot 2.5 \mathrm{~mm}$ for wall shear stress and pressure sensor placement in the model. Best information about the flow is obtained by hot-film and pressure sensors being placed in close proximity. Electromagnetic disturbances of the weak signals from the pressure sensor can be avoided by early amplification and optional digitalization. As a consequence a very compact and waterproof sensor system combining both types of sensor together with integrated signal conversion is required.

\section{STATE OF THE ART}

A huge range of fluidic pressure and direct or indirect shear stress sensors have been developed. Pressure sensors are available based on different sensor principles (i.e. piezoelectric, piezoresistive, capacitive), applicable to various pressure ranges and with different geometric dimensions. A wall shear stress measurement may be realized using a floating element, a fence or heated wire transducing principles [5] [6] [7]. The measurement of pressure and wall shear stress in one miniaturized device is possible but research activities on such combinations are quite limited. Kälvesten et al. [8] designed a sensor system for turbulent measurements in gas flows on a silicon-on-insulator (SOI) substrate. The piezoresistive pressure sensor contains a polysilicon membrane and polysilicon piezoresistors, the shear stress

\footnotetext{
${ }^{1}$ corresponding author: m.schwerter@tu-bs.de
} 
determination is realized by a heated polysilicon filament and a nearby arranged temperature sensing diode. Thermal isolation from the diode as well as the pressure sensor are realized by etched channels filled with polyimide. Bond wires enable electrical connections from the chip to metal conductors.

Another approach is followed by Berns et al. [9] where the diodes are not required. Instead the anemometric principle using the resistance of the heated wire (hot-wire) as feedback is used. Furthermore the thermal isolation of the hot-wire is performed by mounting the wire above a cavity etched in the sensor substrate (silicon). The pressure sensor uses a silicon membrane with implanted piezoresistors etched out of the silicon substrate. For both sensors electrical connections are led by through-silicon-vias to the back side, which enables a back-side contact and thus superseding fragile and potentially flow disturbing bond wires.

The above presented sensor systems seem to be appropriate for measurements of shear stress and pressure at nearly the same point. However, the monolithic integration of both sensors in one chip leads to two main disadvantages:

- Complex sensor system design: Both presented sensor systems are based on SOI-wafers with a multistep manufacturing process. A failure of one sensor part while processing restricts the intended usage of the whole sensor chip.

- Crosstalk: A heating of the pressure sensor due to heat flux from the hot-film sensors - especially in an unbalanced manner - may influence the output signal of the pressure sensor. As shown in [10] the crosstalk in an one-chip solution becomes a problem due the high thermal conductivity of silicon $(142 \mathrm{~W} /(\mathrm{m} \cdot \mathrm{K})$ at $300 \mathrm{~K}$ [11]). Both Kälvesten et al. and Berns et al. integrated head barriers (silicon dioxide (up to $1.4 \mathrm{~W} /(\mathrm{m} \cdot \mathrm{K})$ at $300 \mathrm{~K}[12])$ or polyimide $(\sim 0.1 \mathrm{~W} /(\mathrm{m} \cdot \mathrm{K})$ for spin-on PI2611, recalculated from [13])). However, heat conducting bridges cannot totally be avoided.

Since mentioned approaches are addressing use in gas atmosphere, electrical tracks and functional structures were realized directly on the sensor system's surface without protection. For the use in water environment fragile elements like the hot-wire in the approach of Berns et al. are not suited, especially when particles are present in the water. Finally, much smoother surfaces are required to avoid the appearance of any turbulences due to surface roughness.

To the knowledge of the authors there is no sealed, waterproof sensor system combining hot-film and pressure sensors with amplification electronics integrated into a complete module as presented below.

\section{COMPLETE SENSOR SYSTEM WITH HYBRID INTEGRATION OF SENSORS}

To address the requirements for the use in water environment a hybrid sensor system was developed. The pressure sensor in the form of a rigid chip is flip-chip mounted on the base PCB (printed circuit board). The hot-film sensor in the form of a thin polyimide foil is bonded with epoxy resin on a $200 \mu \mathrm{m}$ thin glass pad by which both sensors are levelled to the same height. The hot-film sensor is mounted with its polyimide substrate facing upwards to provide an electrically passive surface which also protects against the ambient. One end of the flexible polyimide foil is directed down to the base PCB where electrical connection is made by soldering. The PCB holds an amplification circuit for the pressure sensor located nearby on the opposite layer of the PCB, thereby providing shortest electrical connection. An initially small signal in the range of $\mathrm{mV}$ is amplified to values in the range of Volt (up to $4.9 \mathrm{~V}$ ) to make it less susceptible to electromagnetic disturbances. The programmable gain amplifier also compensates offset and temperature drift. The hotfilm signal at comparatively high voltage and power requires no further amplification on the board.

By such hybrid integration of pre-tested single sensors and electronic components the risk of a total system failure due to failures in the sensor production is decreased The assembled and embedded system measures $40 \mathrm{~mm} \cdot 10 \mathrm{~mm} \cdot 2.5 \mathrm{~mm}$ without (shown in Fig. 1) and $60 \mathrm{~mm} \cdot 10 \mathrm{~mm} \cdot 2.5 \mathrm{~mm}$ with on-board digitalization of the pressure sensor signal. Both systems contain two Tappex Microbarb M2 nut inserts for mounting. 


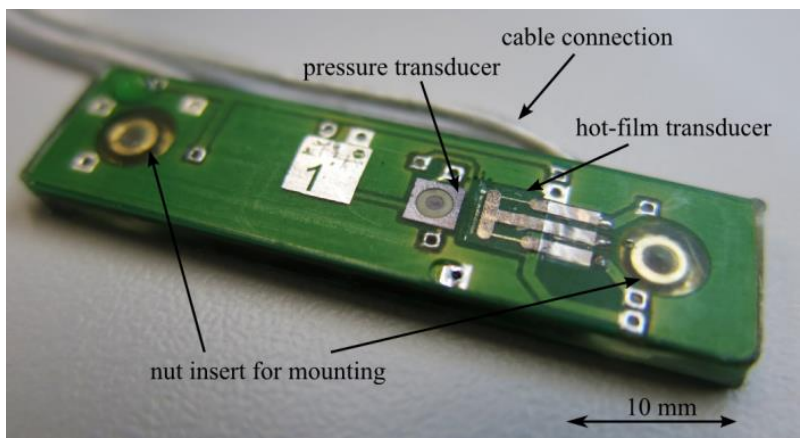

Fig. 1 Embedded sensor system.

\subsection{Pressure sensor}

The pressure sensor design accounts for operation in harsh environment like fast flow of liquids with immersed particles. In contrast to common piezoresistive pressure sensor chips the active elements are arranged at the inside of the sensor. This is achieved by bonding a silicon wafer comprising piezoresistive elements on a glass body by epoxy resin before the silicon final thickness is obtained by unmasked silicon wet-etching. As a consequence, the top silicon surface holds no functional elements and is therefore totally flat and electrically passive. As can be seen from Fig. 2 (a) the remaining silicon is only a thin membrane and the sensors body mainly consists of glass that forms the pressure reference chamber as well as holes for electrical through-glass connection. The membrane is made from an n-doped silicon wafer (100) with four piezoresistors implemented by boron doping. Gold tracks connect these piezoresistors electrically to form a Wheatstone bridge. Finished pressure sensors are shown in Fig. 2 (b).
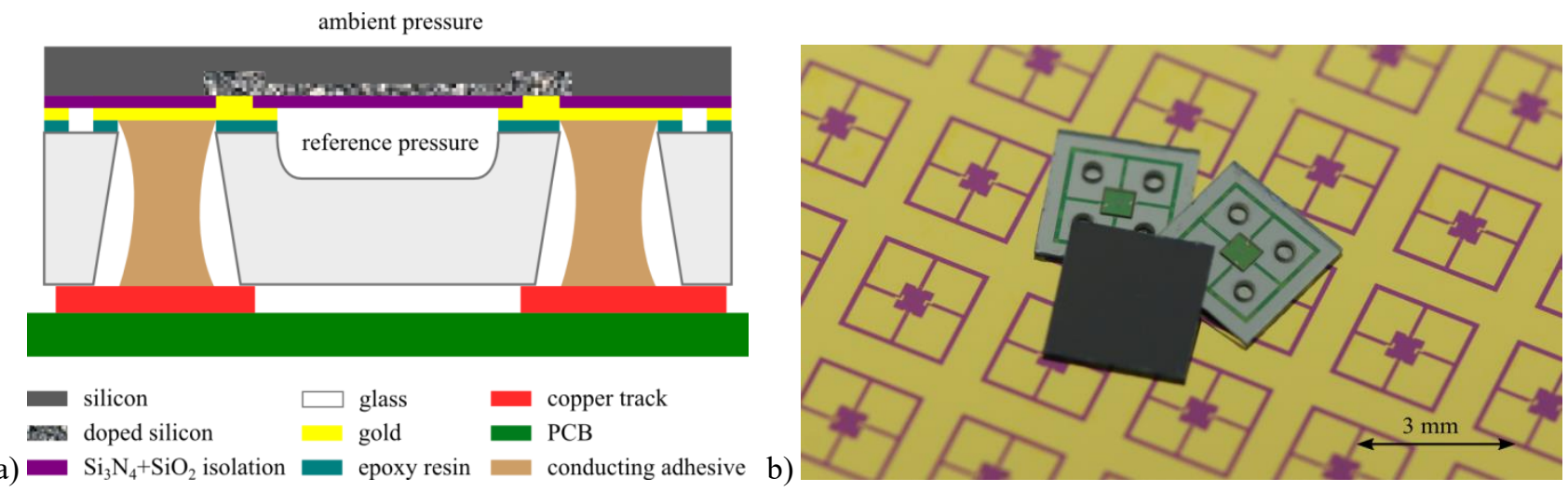

Fig. 2 Simplified schematic cross section of the pressure sensor (a) and three finished sensor chips placed on a silicon wafer with sensing elements prior to the bonding onto the glass body wafer (b). The chip showing the dark (silicon) surface will be in contact with the flow to be measured.

Detailed information about the sensor design, manufacturing and static testing can be found elsewhere [14].

\subsection{Hot-film sensor}

In contrast to a floating element, a fence or a freestanding heated wire the hot-film sensor is robust and its manufacturing is simple. It consists of a thin film filament made of nickel exhibiting a high temperature coefficient of resistance and thus a high sensitivity. The filament is electrically connected by copper tracks. By using spin-on polyimide as foil substrate very thin sensors (down to $7 \mu \mathrm{m}$ ) are realized. Details of the hot-film sensor and its manufacturing in form of an array of 32 sensors have been reported earlier [15]. Here the sensor consists of two hot-film filaments (see Fig. 3) oriented perpendicularly to the flow direction for highest sensitivity. The first filament in the direction of flow is used for anemometric sensing. The second one allows time-of-flight measurements in combination with the first or can substitute the first if that one fails. The thermal shielding of the $5 \mu \mathrm{m}$ thin substrate is low enough to allow an inverse mounting of the sensor with the polyimide foil as protection layer without damping the signal too much. 


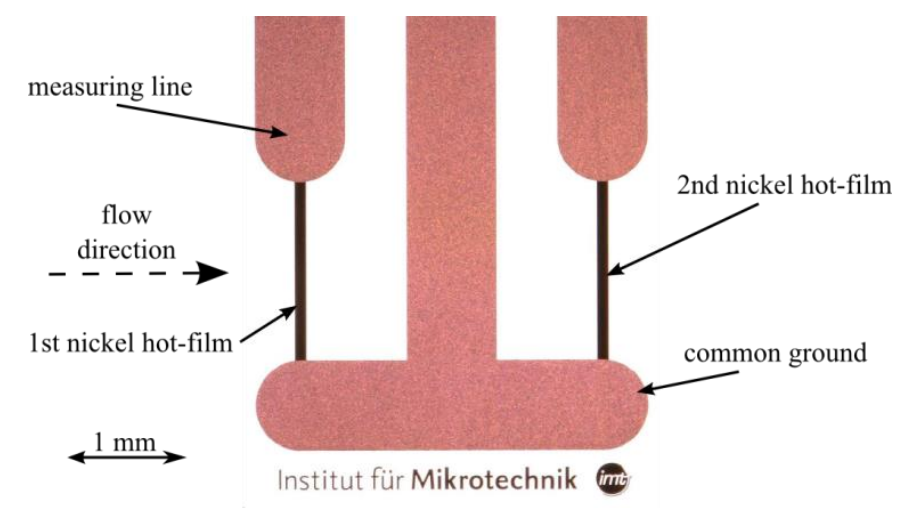

Fig. 3 Micrograph of a hot-film sensor with two filaments (obtained by means of transmitted light microscopy where the transparent foil becomes invisible).

\subsection{Electrical amplification circuit}

A programmable gain amplifier (PGA309 from Texas Instruments) is the core part for on-board amplification. Next to the gain an offset can be corrected. By using an external EEPROM accessible by $\mathrm{I}^{2} \mathrm{C}$ bus up to 17 temperature coefficients can be stored, thus enabling a temperature drift compensation with aid of an internal or external temperature sensor [16]. The pressure sensor is arranged as close as possible (distance $3 \mathrm{~mm}$ ) to the PGA input pins and is connected over an input filter as recommended by the producer [17].

On the $60 \mathrm{~mm}$ long board an additional analog-digital-converter (ADC, part AD7988 from Analog Devices) generates a 16 bit signal which is daisy chain compatible, enabling a serial arrangement of a number of sensor systems over the serial peripheral interface (SPI) [18]. A reference voltage of $2.5 \mathrm{~V}$ (Analog Devices ADR391) is used. The schematic in Fig. 4 gives an overview of the flow of signal processing.

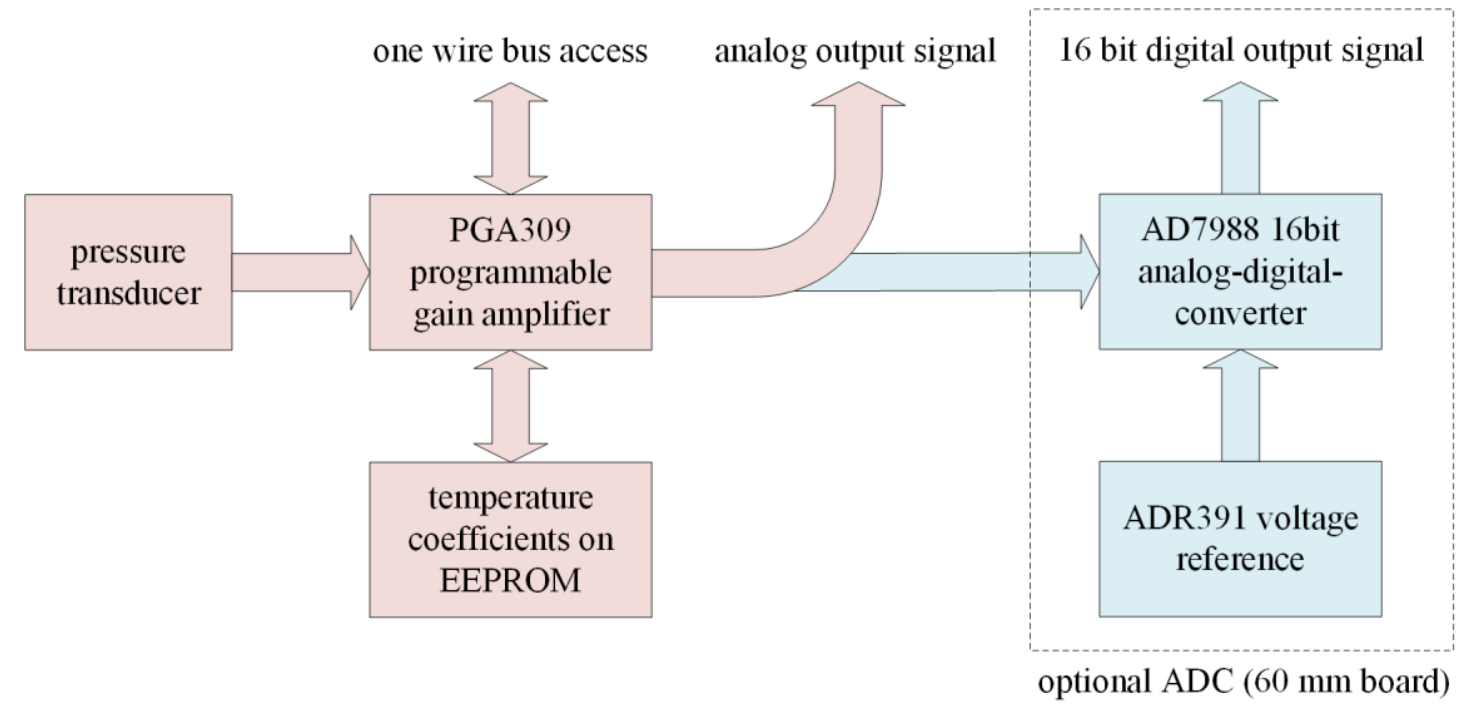

Fig. 4 Schematic of the signal processing flow.

The main goal of the on-board digitalization is the shortening of analog lines and thus minimization of electromagnetic disturbances on the signal. Furthermore the digitalized signal may be directly processed in a digital system (see paragraph 4.2).

The one-wire-bus for PGA309 programming remains accessible after final embedding, so the EEPROM may be programmed prior to or after embedding in epoxy resin. In addition the measured temperature can be read and - instead of compensating with the up to 17 stored temperature values - a more precise compensation may also be performed in the 
external processing system. However, an amplification and rough offset correction is still recommended for higher signal quality to avoid a reduction of the measurement range.

\subsection{Sensor system mounting}

The pressure sensor is electrically connected by first filling the contact holes through the glass body with conductive silver lacquer. In a second step the electrical connection to the board is realized by conductive adhesive (Delo Dualbond IC343). The hot-film sensor is bonded with epoxy resin to the glass pad spacer with the polyimide substrate foil upwards as water protection. The epoxy resin adhesive layer serves also as thermal isolation to the glass pad (thermal conductivities at room temperature: epoxy resin: approx. $0.2 \mathrm{~W} /(\mathrm{m} \cdot \mathrm{K})$ [19], Borofloat 33 glass: $1.08 \mathrm{~W} /(\mathrm{m} \cdot \mathrm{K})$ [20]). The copper tracks are directed to the board and electrically connected by soldering. The complete packaging design with both sensors is shown in Fig. 5.

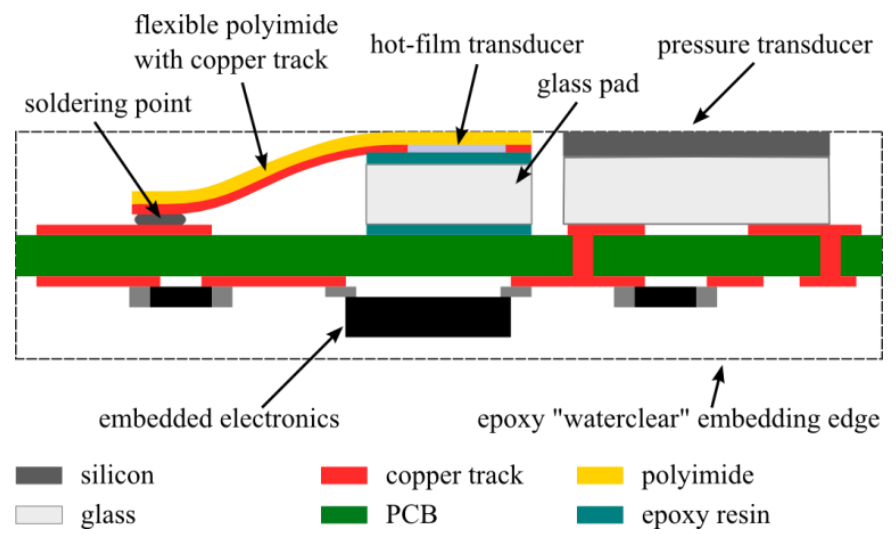

Fig. 5 Hybrid integration of hot-film and pressure sensor with electronics into an epoxy embedding (schematic cross section).

Finally, shielded cables are soldered on the PCB with particular focus on the future position of the cable channels in the casting mold. After final testing the PCB is inserted in a Polytetrafluoroethylene casting mold (see Fig. 6). A sponge-like elastic element on the back side of the PCB presses the sensors flush on the surface of the casting mold after closing. The PCB is embedded in two component epoxy resin "waterclear" (R\&G Faserverbundwerkstoffe GmbH). With its low viscosity $(950+/-100 \mathrm{mPa} \cdot \mathrm{s}$ [21]) it creeps in every cavity of the assembled PCB. In contrast to other tested epoxy resins no self-bending of the module was observed after final hardening. In order to minimize trapped air the epoxy resin is guided to the bottom of the casting mold via a separate channel and slowly rises from the bottom while filling. Thereby air and residual bubbles are pressed out though the opening on top. The embedding with sensors face down enables a smooth module surface on the sensing side not disturbing measurements of the overflowing fluid. 


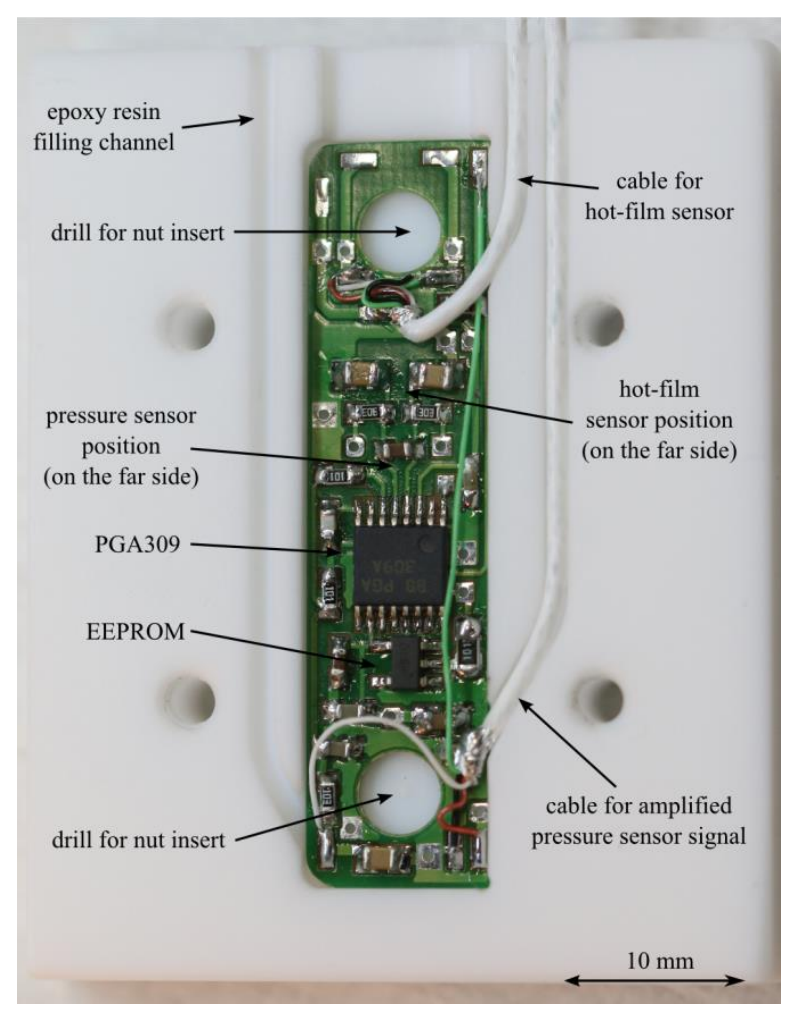

Fig. 6 Sensor system board in the open casting mold. When closing the mold with its lid the sensors are pressed against the surface of the mold. The cables are led through channels; the third channel is used to insert the epoxy resin.

\section{EXTERNAL SIGNAL PROCESSING}

\subsection{Hot-film circuit}

In the filament of the hot-film sensor electrical power causes heating and heat dissipation. Within a small range around the operating point nonlinear contributions can be neglected and the resistance $R_{S}$ as function of the temperature $T_{S}$ of the heated sensor is given by a linear relation:

$R_{S}=R_{0}\left[1+\alpha \cdot\left(T_{S}-T_{0}\right)\right] \quad$ (1)

$R_{0}$ represents the resistance at a reference temperature $T_{0}$ and $\alpha$ the temperature coefficient of resistance [22].

The hot-film sensor is operated in the constant temperature anemometry mode, which means that $T_{S}$ is kept constant. Since variation of the adjacent flow leads to a variation of the heat dissipation the power has to be controlled to stabilize the temperature. Therefore the hot-film is part of a comparatively low ohmic Wheatstone bridge in which the input power of the hot-film is closed loop controlled through the resistance of a potentiometer in the hot-film opposite path in the bridge. With increasing input power to the Wheatstone bridge the hot-film heated temperature and resistance increase. The power is increased until the voltage drop over the hot-film equals the voltage drop over the potentiometer (balanced bridge). The power is controlled by continuously comparing both voltage drops with an operational amplifier. As a consequence, the power needed to heat the hot-film correlates with the cooling due to the flow and with the wall shear stress [15].

\subsection{Multi-sensor digital FPGA based signal processing}

An on-board digitalization of the pressure signal as realized on the $60 \mathrm{~mm}$ long board enables the direct signal processing in digital circuits without being dependent on subsequent external digitalization. Further in daisy-chain mode the amount of cables needed to be installed in the test object for read-out of multiple sensor systems may be strongly reduced. The digital pressure signal from the ADC is accessible via SPI. Even though a simple read-out circuit based on a microcontroller may be used to receive the pressure values we implemented a highly capable logic based circuit in a 
field programmable gate array (FPGA) in order to read out the pressure sensors of several sensor systems simultaneously in real-time. Especially the parallel processing of several implemented processes at the same time in one FPGA allows a fast parallel readout of several sensor systems and also further real-time processing. Here we used the FPGA evaluation board "Nexys 4" by Digilent with Xilinx Artix 7 FPGA (clocked with $100 \mathrm{MHz}$ ). The SPI-access is controlled simultaneously for all pressure sensors in one process. The 16 bit digitalized pressure data from each sensor system can be received with a repetition rate of $50 \mathrm{kHz}$. With the high capability of the FPGA a parallel real-time averaging for all sensor systems may be implemented. If 50 subsequent data points for each sensor are averaged a readout of mean values at $1 \mathrm{kHz}$ is still possible. The averaged data of all sensor systems are collected and send via USB-UART bridge to a computer. Each data point with 16 bit user data is spread over four bytes to add bits determining the data order and the corresponding sensor system number, additionally a start byte indicates the beginning of the data frame.

We successfully performed tests with four parallel sensor systems with plenty of FPGA resources still available (e.g. approximately $1 \%$ usage of slice registers and slice lookup-tables). Therefore, more sensor systems may be connected to the FPGA by SPI and the further processing/averaging processes may be multiplied but limited by the available input/output ports (especially on the evaluation board since many ports are already routed to specific hardware) and the serial UART port. With four sensor systems and $1 \mathrm{kHz}$ data rate, 17 bytes are transmitted every $1 \mathrm{~ms}$, which requires a data rate of at least 170 kilobaud. A data rate of one megabaud was successfully tested (enabling the data transfer of at least 20 sensor systems). The port limits are simply decreased by self-designing an FPGA board instead of using the evaluation board. With common ADC clocking and only two individual signal lines per sensor system (serial data and one-wire configuration/temperature read-out) approx. 100 sensor systems may be read-out by one XC7A100T1CSG324C FPGA with 210 input/output user ports [23] as it is used on the "Nexys 4" board [24].

The sensor data are processed, visualized and stored on a Microsoft Windows computer by either a LabView program or a self-scripted (c\# language using the .net framework) Windows program. Incoming UART-data are assembled to 16 bit data signal arrays. Due to the UART input buffer the data loses its real-time quality but may still be visualized and stored for test evaluation. In contrast, for real time control (for example closed loop control) the data may be directly sent from the FPGA to the controller.

As an alternative the $40 \mathrm{~mm}$ long sensor system board outputs an analog voltage $(0.1 \mathrm{~V}$ to $4.9 \mathrm{~V})$ representing the measured pressure as configured within the PGA309. The voltage may be digitalized with a wide range of devices; for water tunnel measurements a National Instruments NI 9220 data acquisition system is chosen owing to its high performance (16 bit resolution, $100 \mathrm{kS} / \mathrm{s}$ ). The further data processing is performed in LabView.

\section{SENSOR CALIBRATION AND TEST}

The sensor system was tested with regard to its future operation in the water tunnel. Tests include the pressure and hotfilm sensor operation in the embedded state as well as benefits gained by on-board-digitalization. Static tests and confirmation of the functionality for both sensors as single devices were reported earlier [14] [15].

\subsection{Embedded pressure sensor}

Each sensor system is statically calibrated after embedding in a temperature-pressure reference chamber as described earlier [25]. Prior to the calibration a fast pre-calibration is performed for setting the PGA309 in the desired range (for a fixed temperature). After the main calibration with temperatures of $25-40{ }^{\circ} \mathrm{C}$ and absolute pressures between 1 and 3 bar (100 pressure/temperature/output voltage pairs) calibration coefficients $p_{X Y}$ for a polynomial equation in the following form are determined:

$p(t, U)=p_{00}+p_{10} \cdot t+p_{01} \cdot U+p_{20} \cdot t^{2}+p_{11} \cdot t \cdot U+p_{02} \cdot U^{2}$

$t$ represents the PGA309 temperature value in ${ }^{\circ} \mathrm{C}, U$ is the sensor system output voltage in $\mathrm{V}$ and $p(t, U)$ represents the corresponding pressure in bar.

Exemplary values for two fabricated sensor systems are given in Table 1. Using these values the pressure calculation and temperature correction may be performed either externally or by reconfiguring the PGA309 and writing the temperaturedependent coefficients on the associated EEPROM. 
Table 1. Regression coefficients for two sensor systems.

\begin{tabular}{|c|c|c|c|c|c|c|}
\hline Sensor system & p00 & $\mathbf{p}_{10}$ & $\mathbf{p}_{\mathbf{1}}$ & $\mathbf{p}_{\mathbf{2 0}}$ & $\mathbf{p}_{11}$ & $\mathbf{p}_{\mathbf{0 2}}$ \\
\hline 1 & $-1,424$ & 0,03572 & 0,7213 & $-0,0004141$ & $-0,0002603$ & $-0,001008$ \\
\hline 2 & $-1,458$ & 0,02719 & 0,7054 & $-0,0002088$ & $-0,0001634$ & $-0,00115$ \\
\hline
\end{tabular}

The dynamic sensor behavior is investigated by applying a simultaneous air pressure step on both the embedded pressure sensor and a high frequency reference sensor (Kulite XTL-206-190M-3.5BARSG) with a frequency range specified up to $10 \mathrm{kHz}$ with security shielding (grid for protection against touch, particles and other influences). The sensor system is clamped in a holder in which it can be pressurized. The test setup is shown in Fig. 7. The inverse transfer function method [26] calculates the ratio of amplitudes and phase shift for two sensors to be compared, which allows an estimation of the dynamic sensor response relative to a reference sensor. Inverse transfer functions $1 / \mathrm{H}$ for different pressure steps calculated with measured signals of both sensors are shown in Fig. 8. For up to $1 \mathrm{kHz}$ the amplitude ratio remains at around 1 and practically no phase shift is observed up to $1 \mathrm{kHz}$ but above fluctuations occure.

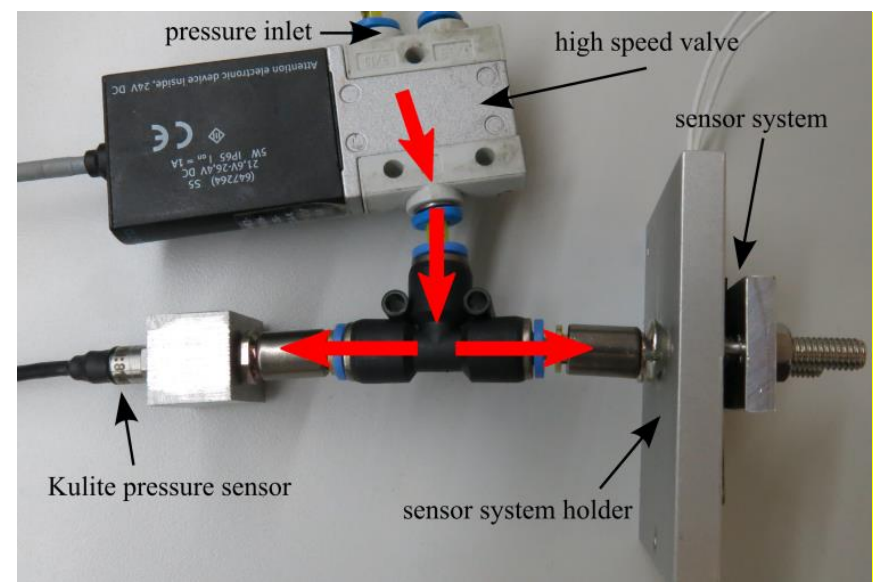

Fig. 7 Test setup for the dynamic pressure sensor test in air. The bold, red arrows indicate the pressure step propagation. 


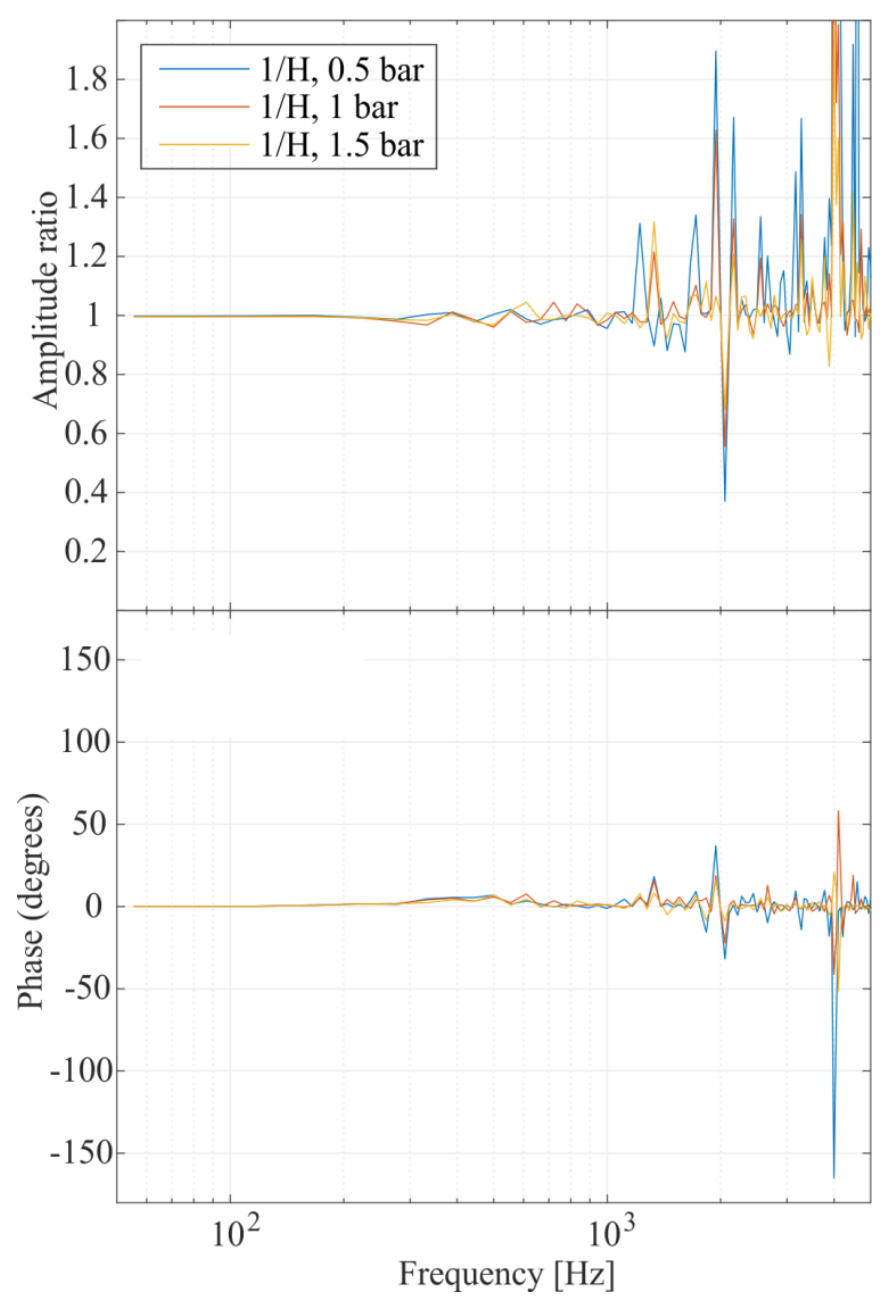

Fig. 8 Amplitude ratio and phase given by the inverse transfer function $1 / \mathrm{H}$ for three pressure steps (measured in air).

\subsection{Embedded hot-film sensor}

With the flipped hot-film sensor the heat has to permeate the polyimide substrate foil. This affects the dynamic sensor response because damping results from the heat capacity and heat resistance of the foil. In order to quantify this effect the hot-film sensor is also characterized using the inverse transfer function method by applying a step air flow on the embedded hot-film sensor and on an unprotected reference sensor. Both sensors are connected to hot-film bridges and are operated in the constant temperature mode (hot-film temperature set to $90{ }^{\circ} \mathrm{C}$ ).

The amplitude ratio and phase shift (data not shown) remain at 1 or $0^{\circ}$ respectively until $2 \mathrm{kHz}$. At $4 \mathrm{kHz}$ the amplitude ratio increases to 1.1 and the phase shifts about approx. $5^{\circ}$.

\subsection{Simultaneous measurements in water}

Finally, a functional demonstration is performed by inserting the sensor system in a tube connected to a water supply. Initially the outlet of the tube is closed (see Fig. 9 a). Water covers the top of the sensor system and thus both sensors (Fig. 9 b). As it can be seen in Fig. 10 the pressure $p_{w}$ of the water slowly increases while water does not flow (flow velocity $v_{w}=0$ ). When suddenly opening the outlet at time zero (see Fig. 9 c) $p_{w}$ drops and $v_{w}$ increases. Simultaneous oscillations in opposing directions occur within both sensor signals (Fig. 10, positive time range) and vanish after reaching a quasi-static state. 
Prior to this test the sensor system was kept in water for 24 hours and tested before and afterwards to verify that it operates correctly in water.

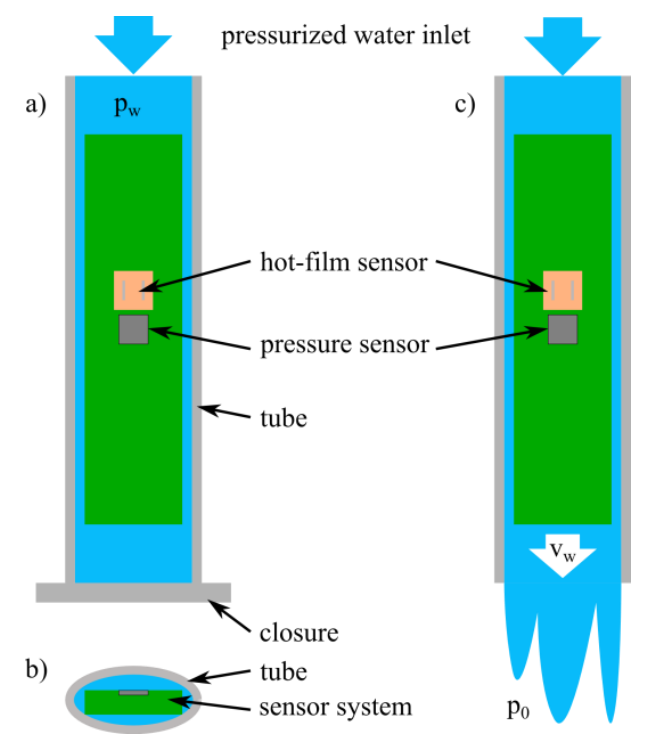

Fig. 9 Schematic of the combined sensor system demonstration. (a) tube with initially closed outlet, (b) cross section of the tube with the inserted sensor system, (c) opened outlet situation.

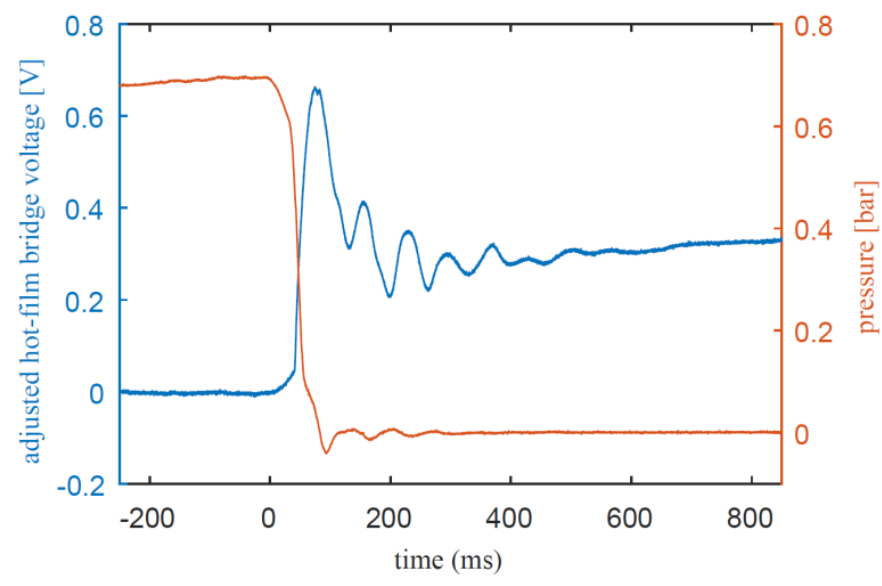

Fig. 10 Sensor signal of the pressure relative to atmosphere pressure (red) and hot-film sensor (blue) in an initially closed water tube which is suddenly opened (at the time 0 ). The hot-film sensor signal is given by the bridge output voltage adjusted by the idle value (at $\left.v_{w}=0\right)$ and $1^{\text {st }}$ order low pass filtered (16 Hz cut-off frequency).

\section{CONCLUSION AND OUTLOOK}

A fully integrated sensor system with pressure and hot-film sensor as well as embedded electronics was designed and realized as a waterproof package allowing direct water contact of the sensor elements. The pressure sensor is designed without functional structures on its top surface, enabling exposure to the fluid. The foil-based hot-film sensor is inversely mounted to protect the heated element and also allow exposure to the fluid and floating particles. Both sensors were tested under dynamic conditions and are fast enough to capture frequencies up to the $\mathrm{kHz}$ range, which is sufficient for the intended measurements in water with up to $300 \mathrm{~Hz}$ [2]. The simultaneous reaction to a flow stimulus of both sensors could be demonstrated with a sensor system integrated in a water tube. Combined sensor systems are now available for the upcoming measurements in a water tunnel where they can give new insights to instabilities occurring at high Reynolds number flows at the surface of wing models. 
Since the SPI of the ADC (AD7988) is daisy chain capable [18] the sensor systems may be serially read-out sensor by sensor in the chain. Thus the number of cables to the read-out circuit (FPGA) is strongly reduced. A connection with board-to-board miniature connectors is already successfully tested. Care should be taken regarding the timing since the sensor systems are read-out one after another and not parallel. The protection of the hot-film sensor may be further optimized in future work: Although the inverted sensor mounting with the polyimide substrate as protection layer enables a simple but sufficient sensor protection the thickness and thus the damping of the protection shall be reduced. As alternate to the polyimide foil a thin film of silicon carbide with suffcient thermal conductivity [27], hardness [28] and chemical resistance (i.e. water vapor resistance see [29]) seems promising.

Future work will focus on a fully flexible sensor system fabricated on a foil substrate. The size of the rigid pressure sensor will be even further reduced down to about $2.2 \mathrm{~mm}^{2}$ to enable the integration without affecting the bendability of the foil. Further the replacement of the adhesive bonding by an anodic or solid-liquid-interdiffusion bonding increases the application temperature of the sensor, enabling a replacement of the conductive adhesive for electrical connection by solder.

\section{ACKNOWLEDGMENT}

This work is founded by the German Research Foundation (DFG) in the framework of the collaborative research center (CRC) 880 "Fundamentals of High-Lift for Future Commercial Aircraft". We gratefully thank Richard Semaan and Yosef El Sayed from the Institute of Fluid Mechanics, TU Braunschweig, for supporting us with the high speed valve and useful hints for signal analysis.

\section{REFERENCES}

[1] International Air Transport Association (IATA), IATA Air Passenger Forecast Shows Dip in Long-Term Demand, Total passengers set to double to 7 billion by 2034, URL: http://www.iata.org/pressroom/pr/Pages/201511-26-01.aspx (accessed on 25.04.2016).

[2] TU Braunschweig Campus Forschungsflughafen, SFB 880 - Fundamentals of high-lift for future commercial aircraft, Biennial Report, CFF Forschungsbericht 2015-04, Braunschweig, 2015.

[3] R. Radespiel, W. Heinze, SFB 880: fundamentals of high lift for future commercial aircraft, CEAS Aeronautical Journal 5 (2014), 3, pp. 239-251, http://dx.doi.org/10.1007/s13272-014-0103-6.

[4] T. Beutel, S. Sattler, Y. El Sayed, M. Schwerter, M. Zander, S. Büttgenbach, M. Leester-Schädel, R. Radespiel, M. Sinapius, P. Wierach, Design of a high-lift experiment in water including active flow control, Smart Materials and Structures 23 (2014), 7, http://dx.doi.org/10.1088/0964-1726/23/7/077004.

[5] L. Löfdahl, M. Gad-el-Hak, MEMS-based pressure and shear stress sensors for turbulent flows, Measurement Science and Technology 10 (1999), 8, pp. 665-686, http://dx.doi.org/10.1088/0957-0233/10/8/302.

[6] T. von Papen, U. Buder, H. D. Ngo, E. Obermeier, A second generation MEMS surface fence sensor for high resolution wall shear stress measurement, Sensors and Actuators A: Physical 113 (2004), 2, pp. 151-155, http://dx.doi.org/10.1016/j.sna.2004.01.058.

[7] M. Sheplak, L. Cattafesta, T. Nishida, C. McGinley, MEMS Shear Stress Sensors: Promise and Progress: 24th AIAA Aerodynamic Measurement Technology and Ground Testing Conference, 2004, http://dx.doi.org/10.2514/6.2004-2606.

[8] E. Kälvesten, C. Vieider, L. Löfdahl, G. Stemme, An integrated pressure-flow sensor for correlation measurements in turbulent gas flows, Sensors and Actuators A: Physical 52 (1996), 1-3, pp. 51-58, http://dx.doi.org/10.1016/0924-4247(96)80125-7.

[9] A. Berns, U. Buder, E. Obermeier, X. Wang, J. Domhardt, J. Leuckert, W. Nitsche, AeroMEMS pressure sensor with integrated wall hot-wire: Proceedings of 2008 IEEE Sensors, 2008, http://dx.doi.org/10.1109/ICSENS.2008.4716746.

[10] T. Beutel, A. Holle, M. Leester-Schädel, S. Büttgenbach, Untersuchungen zur Kombination von Heißfilmen und piezoresistiver Drucksensorik auf einem Chip. MikroSystemTechnik Kongress 2011 Proceedings, 2011. 
[11] H. R. Shanks, P. D. Maycock, P. H. Sidles, G. C. Danielson, Thermal Conductivity of Silicon from 300 to $1400^{\circ}$ K, Phys. Rev. 130 (1963), 5, pp. 1743-1748, http://dx.doi.org/10.1103/PhysRev.130.1743.

[12] M. B. Kleiner, S. A. Kuhn, W. Weber, Thermal conductivity measurements of thin silicon dioxide films in integrated circuits, IEEE Transactions on Electron Devices 43 (1996), 9, pp. 1602-1609, http://dx.doi.org/10.1109/16.535354.

[13] HD MicroSystems, PI 2600 Series - Low Stress Applications, Product Bulletin, URL: http://www.dupont.com/content/dam/dupont/products-and-services/electronic-and-electricalmaterials/semiconductor-fabrication-and-packaging-materials/documents/PI-2600_ProcessGuide.pdf (accessed on 25.10.2016).

[14] M. Schwerter, D. Gräbner, L. Hecht, A. Vierheller, M. Leester-Schädel, A. Dietzel, Surface-Passive Pressure Sensor by Femtosecond Laser Glass Structuring for Flip-Chip-in-Foil Integration, Journal of Microelectromechanical Systems 25 (2016), 3, pp. 517-523, http://dx.doi.org/10.1109/JMEMS.2016.2539202.

[15] M. Schwerter, T. Beutel, M. Leester-Schädel, S. Büttgenbach, A. Dietzel, Flexible hot-film anemometer arrays on curved structures for active flow control on airplane wings, Microsystem Technologies 20 (2014), 4, pp. 821-829, http://dx.doi.org/10.1007/s00542-013-2054-y.

[16] Texas Instruments Incorporated, PGA309 Datasheet, Voltage Output Programmable Sensor Conditioner, URL: http://www.ti.com/lit/ds/symlink/pga309.pdf (accessed on 25.10.2016).

[17] Texas Instruments Incorporated, PGA309 User's Guide, Voltage Output Programmable Sensor Conditioner, URL: http://www.ti.com/lit/ug/sbou024b/sbou024b.pdf (accessed on 25.10.2016).

[18] Analog Devices, Data Sheet AD7988-1/AD7988-5, 16-Bit Lower Power PulSAR ADCs in MSOP/LFCSP, URL: http://www.analog.com/media/en/technical-documentation/data-sheets/AD7988-1_7988-5.pdf (accessed on 25.04.2016).

[19] K. W. Garrett, H. M. Rosenberg, The thermal conductivity of epoxy-resin / powder composite materials, Journal of Physics D: Applied Physics 7 (1974), 9, pp. 1247-1258, http://stacks.iop.org/0022-3727/7/i=9/a=311.

[20] SCHOTT Technical Glass Solutions GmbH, SCHOTT BOROFLOAT ${ }^{\circledR}$ 33, The versatile floated borosilicate glass-with an infinite number of applications, URL: http://psec.uchicago.edu/glass/borofloat_33_e.pdf.

[21] R\&G Faserverbundwerkstoffe, Technical Data Epoxy casting resin "waterclear", URL: http://www.ezentrumbilder.de/rg/pdf/td_en_Epoxy\%20casting\%20resin\%20waterclear.pdf (accessed on 25.04.2016).

[22] T. Beutel, M. Leester-Schädel, A. Dietzel, Manufacturing of flexible micro hot-film probes for aeronautical purposes, Microelectronic Engineering 111 (2013), pp. 238-241, http://dx.doi.org/10.1016/j.mee.2013.03.173.

[23] XILINX $\quad$ Inc., $\quad 7 \quad$ Series $\quad$ FPGAs URL: http://www.xilinx.com/support/documentation/data_sheets/ds180_7Series_Overview.pdf (accessed on 28.6.16).

[24] Digilent, Nexys $4^{\mathrm{TM}} \quad$ FPGA Board Reference URL: https://reference.digilentinc.com/_media/nexys:nexys4:nexys4_rm.pdf (accessed on 28.6.16).

[25] T. Beutel, M. Leester-Schädel, S. Büttgenbach, Design and evaluation process of a robust pressure sensor for measurements in boundary layers of liquid fluids, Microsystem Technologies 18 (2012), 7, pp. 893-903, http://dx.doi.org/10.1007/s00542-011-1404-x.

[26] R. Semaan, P. Scholz, Pressure correction schemes and the use of the Wiener deconvolution method in pneumatic systems with short tubes, Experiments in Fluids 53 (2012), 3, pp. 829-837, http://dx.doi.org/10.1007/s00348-012$1332-2$.

[27] P. M. Sarro, Silicon carbide as a new MEMS technology, Sensors and Actuators A: Physical 82 (2000), 1-3, pp. 210-218, http://dx.doi.org/10.1016/S0924-4247(99)00335-0. 
[28] T. Frischmuth, M. Schneider, D. Maurer, T. Grille, U. Schmid, Inductively-coupled plasma-enhanced chemical vapour deposition of hydrogenated amorphous silicon carbide thin films for MEMS, Sensors and Actuators A: Physical 247 (2016), pp. 647-655, http://dx.doi.org/10.1016/j.sna.2016.05.042.

[29] W. Daves, A. Krauss, N. Behnel, V. Häublein, A. Bauer, L. Frey, Amorphous silicon carbide thin films (a-SiC:H) deposited by plasma-enhanced chemical vapor deposition as protective coatings for harsh environment applications, Thin Solid Films 519 (2011), 18, pp. 5892-5898, http://dx.doi.org/10.1016/j.tsf.2011.02.089. 